\title{
Cloning and functions of the HBxAg-binding protein XBP1
}

\author{
WANHU FAN, JUN CHENG, SHULIN ZHANG and XIAOJING LIU \\ Department of Infectious Diseases, The First Affiliated Hospital of Medical School, \\ Xi'an Jiaotong University, Xi'an 710061, P.R. China
}

Received June 22, 2012; Accepted October 24, 2012

DOI: $10.3892 / \mathrm{mmr} .2012 .1232$

\begin{abstract}
In the present study the hepatitis B virus $\mathrm{X}$ antigen binding protein 1 (XBP1) was cloned by inducing its expression, and its subcellular localization and function were examined. Total RNA was extracted from HepG2 cells and $X B P 1$ was amplified using reverse transcription polymerase chain reaction (RT-PCR), followed by restriction enzyme digestion of the pGBKT7 yeast plasmid and identification by enzyme digestion. The plasmid was transformed into AH109 yeast via the lithium acetate method and protein extracts were prepared. XBP1 protein expression in the eukaryotic cells was determined using polyacrylamide gel electrophoresis and western blot analysis. The gene encoding the XBP1-binding protein was screened in liver cells using yeast two-hybrid technology. We transfected a human hepatocellular carcinoma cell line and observed the intracellular localization of the gene expression protein using a fluorescence microscope, followed by prokaryotic expression and XBPl gene identification. A 921-bp XBPl gene fragment was obtained via RT-PCR amplification and 20 proteins with known functions that interact with XBP1 were screened, including metallothionein, smooth muscle cell-related protein, asialoglycoprotein receptor, pyruvate dehydrogenase kinase 1 and a sequence with unknown functions. A green fluorescent protein expression plasmid pEGFP-C1-XBP1 of XBP1 was constructed successfully and its expression protein was localized in the cytoplasm. A $56-\mathrm{kDa}$ recombinant protein was successfully obtained via prokaryotic expression and was demonstrated to have good specificity using western blot analysis. The XBPl gene, which expresses the XBP1 protein, is located in the cytoplasm and plays a role in the intracellular structure, cell growth, intracellular metabolism and signal transduction pathway, as well as DNA duplication, transcription, recombination and repair.
\end{abstract}

Correspondence to: Professor Jun Cheng, Department of Infectious Diseases, The First Affiliated Hospital of Medical School, Xi'an Jiaotong University, 277 Yantaxi Road, Yanta, Xi'an 710061, P.R. China

E-mail: chengjuncn@yeah.net

Key words: hepatitis B virus, antigen-binding protein 1, yeast two-hybrid

\section{Introduction}

The hepatitis B virus (HBV) has spread worldwide. Existing data indicate that more than one million patients succumb to hepatocirrhosis and liver cancer annually. A marked correlation has been observed between prevalence of HBV carriers and incidence of hepatocellular carcinoma $(1,2)$.

The HBV genome is a partially double-stranded cyclic DNA. The $\mathrm{X}$-open reading frame (ORF) that encodes the $\mathrm{X}$ protein is located between 1,376 and 1,837 nt near the cohesive end of the viral genome and the $H B x$ gene is the smallest gene in the HBV genome. The encoded X protein is composed of 154 amino acids with a relative molecular mass of $17 \mathrm{kDa}$ and is mainly located in the cytoplasm, with a small amount located in the nucleus $(3,4)$. As a multipurpose viral protein, $\mathrm{HBx}$ interacts with a number of proteins, including cytoplasmic proteins, such as Jak1, PKC binding protein and Caspase-3; nuclear proteins, such as TFIIB, RXR and TBP; and proteins shuttling between the cytoplasm and cell nucleus, such as Smad4 and Tat-binding protein (5). HBx has an extensive gene transcription regulation function and interacts with numerous proteins in the host cell to regulate gene expression and cell protein function, thereby affecting the biological functions of the virus, including self-duplication, signal transduction of the host cell, cell multiplication, carcinogenesis and differentiation and apoptosis (6-10). The $H B V X$ gene and HBx protein are both involved in HBV infection, duplication, pathogenesis and possibly carcinogenesis and play an important role in the course of chronic infection. Clarifying the structure and function of XBP1 and its effect on HBV-induced liver injury facilitates the investigation of the function and mechanism of action of the $X$ gene and HBx protein and may contribute to the control and treatment of chronic HBV infection. The present study lays a foundation for additional understanding of chronic HBV infection and the pathogenesis of primary liver cancer.

\section{Materials and methods}

XBP1 gene amplification. The study was approved by the ethics committee of Beijing Ditan Hospital, Beijing, China. Total RNA extraction from HepG2 cells and cDNA synthesis was performed according to a previously described method (11). Sequence-specific primers were designed for $X B P 1$ gene amplification via PCR according to the full-length $X B P 1$ gene: sense primer 5'-GCCGAATTCATGGCCAAGGACTTTCAA GA-3' and antisense primer 5'-TAAGGATCCTCAGGCCACC 
TCGCCGGTGGC-3'. The XBPl gene was amplified via PCR and the target DNA fragments were subsequently recovered and ligated into the pGEM-T vector (Promega, Madison, WI, USA), followed by double enzyme digestion and sequencing.

Recombinant bait vector construction and self-activation detection. The pGBKT7 yeast plasmid and the XBP1 gene were digested with $E c o$ RI/SalI, purified and ligated overnight using T4 DNA ligase at $16^{\circ} \mathrm{C}$, transformed into E. coli DH5 $\alpha$ and screened. The plasmid was extracted and identified via EcoRI and XhoI double enzyme digestion and sequencing and the recombinant was named pGBKT7-XBP1. The pGBKT7-XBP1 vector was transformed into the AH109 yeast cells using the lithium acetate method according to the manufacturer's instructions (Clontech Corporation, Mountain View, CA, USA) and then plated on the synthetic dropout medium-tryptophan (SD/-Trp) for screening. Colonies ( $>2 \mathrm{~mm}$ in diameter) were identified via PCR, whereas $100 \mu 1$ stock solution was directly spread onto the SD/-Trp/-His/-Ade culture medium with kanamycin for the self-activation experiment.

Western blot analysis. Protein extract was prepared using the urea/sodium dodecyl sulfate (SDS) method (12) and stained using diaminobenzidine tetrachloride with anti-c-Myc monoclonal antibodies diluted to 1:100 as the primary antibody and horseradish peroxidase (HRP)-labeled goat anti-mouse IgG diluted to $1: 2,500$ as the secondary antibody (13).

Liver cDNA library screening. Several AH109 yeast colonies ( $>2 \mathrm{~mm}$ in diameter) containing the pGBKT7-XBP1 plasmid were selected from the SD/-Trp culture medium, inoculated into $\mathrm{SD} /$-Trp liquid culture medium, agitated at $30^{\circ} \mathrm{C}$ and $250 \mathrm{rpm}$ for $16-24 \mathrm{~h}$ and mated with $400 \mu \mathrm{l}$ of yeast cells in the liver cell library in $50 \mathrm{ml}$ of $2 \mathrm{X}$ yeast peptone dextrose adenine (YPDA) at $30^{\circ} \mathrm{C}$ and further agitated at $30-45 \mathrm{rpm}$ for $18-24 \mathrm{~h}$ when the $\mathrm{OD}_{600}$ reached 0.8 and 1.0. Clover leaf-shaped diploid cells were observed, resuspended in $10 \mathrm{ml} 0.5 \mathrm{X}$ YPD culture medium, spread on 25 plates with $150 \mathrm{~mm}$ of SD/-Trp/-Leu/-His and 25 plates with D/-Trp/-Leu/-His/-Ade and cultured at $30^{\circ} \mathrm{C}$ until a colony was formed. The monoclonal cells grown on $\mathrm{SD} /-\mathrm{Trp} /-\mathrm{Leu} /$-His/-Ade were spread onto QDO with X- $\alpha$-Gal for streak culture at $30^{\circ} \mathrm{C}$ for $4-8$ days and the blue colony was the positive colony. The plasmid from positive yeast was extracted and transformed into E. coli using electroporation (14), followed by plate cultivation in ampicillin-containing Luria-Bertani culture medium. The plasmid was extracted from the obtained colony, digested with $B g l \mathrm{II}$, sequenced and its homology with sequences in the GenBank database was analyzed.

Subcellular localization of XBP1. The XBP1 primer was designed using the NTI software package. A ' $G$ ' was inserted between the EcoRI site and ATG in this primer via in-frame expression with pEGFP-C1. P1: 5'-GCCGAATTCGATGGC CAAGGACTTTCAAGA-3' EcoRI; P2: 5'-TAAGGATCC TCAGGCCACCTCGCCGGTGGC-3' BamHI. The PCR amplification product was recovered and ligated with the pGEM-T vector for sequencing. The $X B P 1$ and pEGFP-C1 plasmids were extracted, digested with EcoRI and BamHI and the $X B P 1$ and pEGFP-C1 were ligated using ligase and identified via enzyme digestion. Human hepatocellular carcinoma
HepG2 cells were transfected with the purified plasmid and a blank vector was used as the blank control. One day prior to transfection, HepG2 cells were trypsinized, counted and inoculated in a small plate with a diameter of $35 \mathrm{~mm}$ and $50 \%$ of the cells were cohered on the day of transfection. At $48 \mathrm{~h}$ after transfection, images of the HepG2 cells were captured using a digital camera under a fluorescence microscope.

Expression of XBP1 gene in E. coli. The following PCR amplification primers were designed for the $X B P 1$ gene: sense primer 5'-GCCGAATTCATGGCCAAGGACTTTCAAGA-3' and antisense strand primer 5'-TAAGTCGACTCAGGCCA CCTCGCCGGTGGC-3'; the underlined sites are EcoRI and SalI enzyme digestion sites, respectively. The target fragment was recovered following PCR amplification with the pGEMTXBP1 plasmid as the template. The recovered target gene fragment was ligated into the pGEM-T vector using T4 DNA ligase and those with the correct sequence were selected for EcoRI/SalI enzyme digestion, connected to the pET-32a(+) expression plasmid with the same double enzyme digestion for identification. The pET32a(+)XBP1 plasmid identified to be correct was transformed into E. coli BL21 for isopropyld-thiogalactopyranoside (IPTG) and SDS-polyacrylamide gel electrophoresis (PAGE) analysis.

Western blot analysis. Anti-His monoclonal antibody diluted to 1:200 and HRP-IgG diluted to $1: 2,500$ were used as the primary and secondary antibodies, respectively. Based on conventional SDS-PAGE and western blot analysis, membranes were blocked overnight in 5\% dried skimmed milk, incubated with the primary and secondary antibodies, gently agitated at room temperature following the addition of color reagent and exposed to X-rays.

\section{Results}

$X B P 1$ gene amplification. The ORF of the XBP1 gene is $921 \mathrm{nt}$ long and the encoded product is composed of 307 amino acid residues (Fig. 1). Identification through enzyme digestion demonstrated that the pGEM-T-XBP1 plasmid digested with EcoRI and BamHI enzyme had a normal size and was confirmed as a plasmid.

Recombinant bait vector construction and self-activation detection. The pGBKT7-XBP1 plasmid construction was analyzed using the Vector NTI Suite 8.0 software; EcoRV and SalI restriction enzyme digestion sites present in the vector and target fragment were selected for enzyme digestion analysis. Two EcoRV fragments, 7535 and 686 nt, and three SalI fragments, 5664, 1809 and 748 nt, were obtained. The $X B P 1$ sequence amplified via PCR from the positive colonies had a normal size. The colony did not grow on the SD/-AdeTrp-His solid culture medium plate, which indicates that this bait strain had no self-activation phenomenon and subsequent screening by mating may be performed.

Western blot analysis. Following 12.5\% SDS-PAGE of the yeast protein extract, protein expression was detected by western blot analysis (Fig. 2). The established 'bait' vector pGBKT7-XBP1 was transformed into yeast $\mathrm{AH} 109$ and the XBP1 fusion protein 


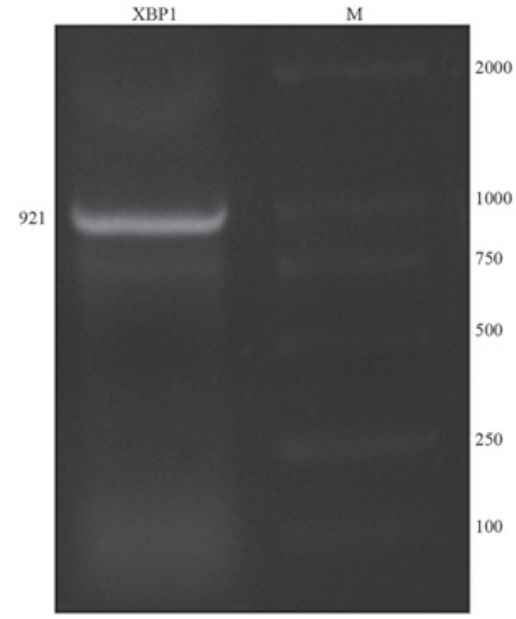

Figure 1. XBP1 gene (921 bp) was amplified by PCR. Target DNA fragments were recovered and ligated into the pGEM-T vector followed by double enzyme digestion with $E c o$ RI and BamHI enzymes to confirm correct amplification of the $X B P 1$ gene was performed. $\mathrm{XBP} 1$, hepatitis $\mathrm{B}$ virus $\mathrm{X}$ antigen binding protein 1 .

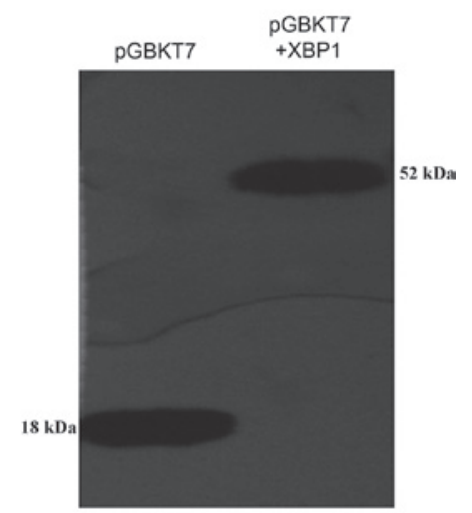

Figure 2. Western blot analysis to confirm stable expression of XBP1 fusion protein in the pGBKT7 vector. Lane 1, vector only (18,200 kDa). Lane 2 , vector + XBP1 fusion protein $(33,770 \mathrm{kDa})$, represented by a band corresponding to $51,970 \mathrm{kDa}$. XBP1, hepatitis B virus $\mathrm{X}$ antigen binding protein 1 .

$\mathbf{A}$

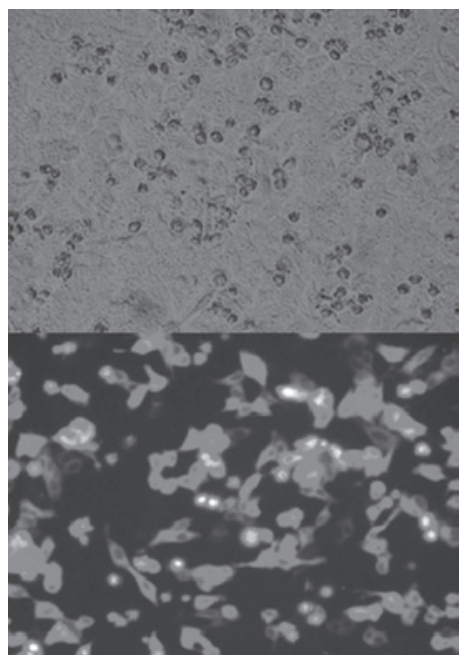

Figure 3. (A) HepG2 cells. (B) Cells successfully tranfected with the pEGFP-XBP1 expression plasmid showed green fluorescence diffused in the cytoplasm under a fluorescent microscope. Magnification, x200. XBP1, hepatitis B virus $\mathrm{X}$ antigen binding protein 1 .

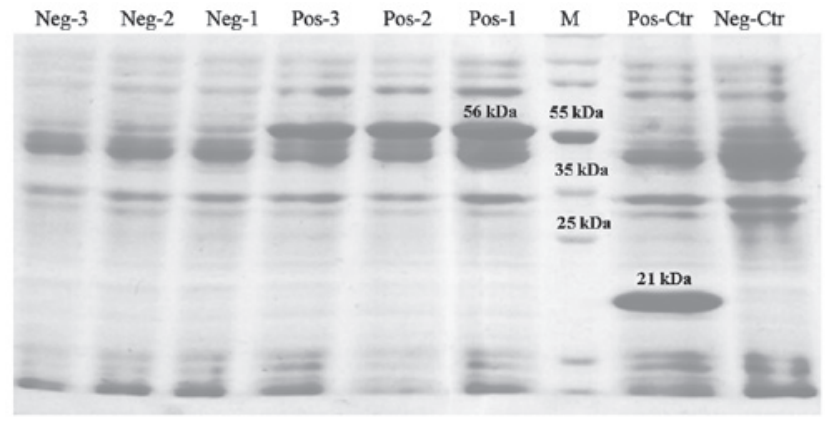

Figure 4. Expression of the XBP1 gene in E. coli. PET32a-XBP1 protein expression was detected by PAGE gel electrophoresis. Neg-3-1, pET-32a(+)XBP1 uninduced group. Pos-3-1, pET-32a(+)-XBP1 induced group. Pos-Ctr and Neg-Ctr, pET-32a(+) empty plasmid induced and uninduced groups, respectively. Negative ctr, the uninduced protein in $E$. coli BL21 was expressed with numerous molecular weights. Positive ctr, the IPTG-induced BL21 protein expression was $\sim 56 \mathrm{kDa}$, the same as the predicted molecular weight of the recombinant protein and inhibited the non-target protein expression.

was stably expressed. The molecular weight of pGBKT7 in the positive control group was 18,200; XBP1, 33,770; and XBP1 fusion protein in the experimental group, 51,970. Western blot analysis demonstrated that the yeast extract transforming the pGBKT7 plasmid and pGBKT7-XBP1 plasmid revealed bands at the corresponding molecular weight and its molecular weight was consistent with the theoretical value.

Liver cDNA library screening. The clone numbers of mated product diluted to 1:1,000 and growing on SD/-Trp culture media could not be counted, and those on SD/-Leu and $\mathrm{SD} /$-Trp-Leu culture media were 187 and 14, respectively. The survival rate of Y18 was calculated at $1.87 \times 10^{6} \mathrm{cfu} / \mathrm{ml}$ (restricted part), whereas that of the diploid was $1.4 \times 10^{5} \mathrm{cfu} / \mathrm{ml}$ and the mating efficiency was $7.5 \%$.

Blue and white screening of the QDO culture medium containing $\mathrm{x}-\alpha$-gal revealed that blue colony was the positive colony. On the D/-Trp/-Leu/-His/-Ade/X- $\alpha$-gal culture medium, 86 positive colonies were screened, 83 yeast plasmids were successfully extracted and 80 yeast plasmids were successfully electrotransformed and cloned into E. coli.

The pACT2 library vector includes two BglII enzyme digestion sites located on both sides of the polyclonal site. The leukocyte library fragment was released with this enzyme digestion and 68 plasmids were identified as correct via $B g l \mathrm{II}$ enzyme digestion.

The cDNA sequencing and homology analysis results indicate that 36 positive clones were selected for sequencing and 20 known protein-encoding genes and 1 gene with unknown function were obtained (Table I).

$X B P 1$ subcellular localization. The XBPI gene was successfully connected to $\mathrm{pEGFP-C1}$ and was identified as correct via enzyme digestion. As shown in Fig. 3, the pEGFP-XBP1 expression plasmid was successfully expressed in HepG2 cells and observation under a fluorescence microscope revealed green fluorescence diffused in the cytoplasm of the transfected cells.

Expression of the XBP1 gene in E. coli. The pcDNA3.1(-)-XBP1 plasmid was successfully constructed and verified to be correct 
Table I. Results of liver cDNA library screening.

\begin{tabular}{|c|c|c|c|}
\hline No. & Coding protein with known homologous sequence & $\begin{array}{l}\text { Homologous } \\
\text { clone number }\end{array}$ & Homology $(\%)$ \\
\hline 1 & Human asialoglycoprotein receptor 1 & 12 & 98 \\
\hline 2 & Human betaine-homocysteine methyltransferase & 1 & 100 \\
\hline 3 & Human solute carrier family 25 & 3 & 100 \\
\hline 4 & Human mitochondrial DNA & 2 & 100 \\
\hline 5 & Human diazepam binding inhibitor & 2 & 100 \\
\hline 6 & Transforming growth factor $\beta 1$ & 1 & 99 \\
\hline 7 & Human cyclic AMP response element binding protein 3 & 1 & 99 \\
\hline 8 & Human retinol-binding protein 4 & 1 & 99 \\
\hline 9 & Human complement factor B & 1 & 99 \\
\hline 10 & Human CD74 molecule, major histocompatibility complex, transcript 3 & 1 & 100 \\
\hline 11 & Human metallothionein $2 \mathrm{~A}$ & 1 & 100 \\
\hline 12 & Human chromosome 10 clone RP11-45D20 & 2 & 98 \\
\hline 13 & Human topoisomerase II $\beta 180-\mathrm{kDa}$ & 1 & 97 \\
\hline 14 & Human serum albumin & 1 & 99 \\
\hline 15 & Human pyruvic dehydrogenase kinase 1 & 1 & 99 \\
\hline 17 & Human serine proteinase inhibitor & 1 & 100 \\
\hline 18 & Smooth muscle cell-related protein & 1 & 99 \\
\hline 19 & Human complement C9 & 1 & 100 \\
\hline 20 & Human CD74 molecule, major histocompatibility complex, transcript 2 & 1 & 100 \\
\hline 21 & Gene 1 with unknown function & 1 & $97-100$ \\
\hline
\end{tabular}

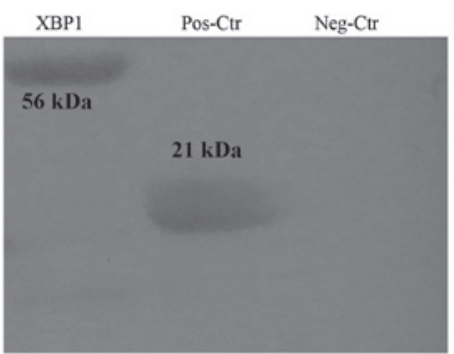

Figure 5. BL21-pET32a(+) and BL21- pET32a(+) XBP1 bacterial proteins were detected by western blot analysis.

via enzyme digestion. The PET32a(+)-XBP1 plasmid was correct under PCR amplification and EcoRI and SalI enzyme digestion. The PET-32a-XBP1 recombinant protein in $E$. coli was detected using $12.5 \%$ PAGE and revealed that the uninduced protein in E. coli BL21 was expressed with numerous different molecular weights. The IPTG-induced BL21 expression protein was $\sim 56 \mathrm{kDa}$, the same as the predicted molecular weight of the recombinant protein and inhibited the non-target protein expression (Fig. 4). BL21-pET32a(+) and BL21-pET32a(+) XBP1 bacterial proteins were detected by western blot analysis and the results indicate marked banding at $21.8 \mathrm{kDa}$ of the former and $55.6 \mathrm{kDa}$ of the latter (Fig. 5).

\section{Discussion}

In the present study, $X B P 1$ was inserted into the pGBKT7 vector and the fusion protein-expressing recombinant plasmid was constructed successfully. The plasmid was then trans- fected into AH109. Western blot analysis (involving the use of anti-myc monoclonal antibody prepared using the hybridoma technique) demonstrated that the fusion protein was expressed, which laid a basis for further screening, as well as the application and development of the protein.

$X B P 1$ binding protein genes in the white blood cell library were screened using yeast two-hybrid assay. Human asialoglycoprotein receptor 1 , human betaine homocysteine methyltransferase, human cAMP responsive element binding protein 3 , human retinol binding protein 4 , human serine proteinase inhibitors, human CD74 molecules, major histocompatibility complex, human complement factor $\mathrm{B}$, human complement C9, human pyruvate dehydrogenase kinase 1, contractile fiber cell associated protein 2 , human topoisomerase II $\beta$ 180-kDa, metallothionein $2 \mathrm{~A}$ and transforming growth factor $\beta 1$ were obtained. These molecules perform important roles, consistent with results of bioinformatics analysis $(15,16)$.

In the present study, 12 homologous clones were screened. ASGPR, the endocytic receptor of a heterologous oligomer, is located on the surface of the cell membrane in the hepatocyte facing the sinusoid. The functional area wherein ASGPR identifies and binds with the lactose residue or acetyl galactosamine residue is called the carbohydrate recognition domain, which includes two subgroups, $\mathrm{H} 1$ and H2. The H1 subgroup is important in identifying ligands and it mediates endocytosis via ASGPR (17). ASGPR identifies and specifically binds with sugar chains that contain a galactose or N2-acetyl galactosamine residue at the end and transports its ligand into the lysosome for degradation via hepatocellular endocytosis. Therefore, the main physiological function of ASGPR is the removal of asialoglycoprotein, apoptotic cells, lipoproteins, etc. (18). 
The serine proteinase inhibitor, a serine proteinase activity regulator, is associated with blood coagulation, fibrinolysis, complement activation, inflammatory reaction and tissue reconstruction process and is involved in the inhibition of tumor invasion and metastasis (19). It performs a transcription regulation function via autophosphorylation (20). Special attention has been given to the correlation between cAMP response element-binding (CREB) and molecular nerve mechanism of learning and memory; CREB promotes the formation of longterm memory among fruit flies, mice and other animals (21).

The main function of the smooth muscle cell-related protein is promoting cell survival, extending cell life, regulating apoptosis, promoting vascular smooth muscle cell (VSMC) proliferation and migration and promoting the mammary stromal differentiation towards myofibroblasts (22). It inhibits cell growth (23) by inhibiting the transcription start site (24), prevents the entry of blood cells into the S phase and directly inhibits the action of pluripotential hematopoietic stem cells.

The indirect enzyme-linked immunosorbent assay for detecting the anti-HBx antibodies in serum was established using the recombinant protein HBx to detect and observe changes in levels of anti-HBx antibodies in the serum of patients with hepatitis B (25). The XBP1 gene was expressed using the $E$. coli system and validated via western blot analysis. This expression is likely to lay the foundation for further studies on the effect of $X$ protein on the immunological function of the host, experiments on further purification of the immunogenic XBP1 protein and polyclonal antibody and provides the basis for clinical examination. The XBPI gene was successfully connected to pEGFP and identified via enzyme digestion. The pEGFP-XBP1 expression plasmid was successfully expressed in HepG2 cells and fluorescence microscopy indicated that green fluorescence was diffused in the cytoplasm of transfected cells, but no green fluorescence protein expression was observed in the cell nucleus. Knowledge of the subcellular localization of XBP1 to the cytoplasm generates additional understanding of gene function. In future steps, the protein is likely to be purified to obtain sufficient high-purity XBP1 protein for animal inoculation and polyclonal and monoclonal antibodies against XBP1 prepared using the hybridoma technology. Once the antibody is available, immunohistochemical research may be performed to clarify the correlation between the protein expression of the gene, its expression mechanism and clinical disease evolution, thereby revealing the biological and medicinal significance of the gene.

Binding proteins screened using yeast two-hybrid technology were classified as follows: i) proteins related to the intracellular structure and cell growth; ii) proteins involved in intracellular metabolism; iii) proteins involved in signal transduction pathways, immunity and other related proteins; and iv) proteins involved in DNA duplication, transcription, recombination and repair. The identification of these binding proteins provides new insight into the biological function of XBP1, HCV pathogenesis and the reason for its malignant transformation. Analysis of the XBP1 indicates that following its intracellular expression, the expression of genes related to cell growth, differentiation, material and energy metabolism, signal transduction and tumorigenesis is increased. The present study indicates that XBP1 may affect numerous systems in vivo, providing new clues as to the role of XBP1 and HBX in pathogenesis.

\section{References}

1. Wang Y, Liu H, Zhou Q and Li X: Analysis of point mutation in site 1896 of HBV precore and its detection in the tissues and serum of HCC patients. World J Gastroenterol 6: 395-397, 2000.

2. Zhuang L, You J, Tang BZ, et al: Preliminary results of Thymosin-a1 versus interferon-treatment in patients with $\mathrm{HBeA}$ $\mathrm{g}$ negative and serum HBV DNA positive chronic hepatitis B. World J Gastroenterol 7: 407-410, 2001.

3. Henkler F, Hoare J, Waseem N, et al: Intracellular localization of the hepatitis B virus HBx protein. J Gen Virol 82: 871-882, 2001.

4. Hoare J, Henkler F, Dowling JJ, et al: Subcellular localization of the X protein of HBV infected hepatocytes. J Med Virol 64: 419-246, 2001.

5. Tang H, Delgermaa L, Huang F, et al: The transcriptional transactivation function of $\mathrm{HBx}$ protein is important for its augmentation role in hepatitis B virus replication. J Virol 79: 5548-5556, 2005.

6. Zhang JL, Zhao WG, Wu KL, et al: Human hepatitis B virus $\mathrm{X}$ protein promotes cell proliferation and inhibits cell apoptosis through interacting with a serine protease Hepsin. Arch Virol 150: 721-741, 2005.

7. Chan DW and Ngi O: Knock-down of hepatitis B virus X protein reduces the tumorigenicity of hepatocellular carcinoma cells. J Pathol 208: 372-380, 2006.

8. Park SG, Chung C, Kang H, Kim JY and Jung G: Up-regulation of cyclin D1 by HBx is mediated by NF-kB2/BCL3 complex through $\kappa \mathrm{B}$ site of cyclin D1 promoter. J Biol Chem 281: 31770-31777, 2006.

9. Chen YB, Yan ML, Gong JP, et al: Establishment of hepatocellular carcinoma multidrug resistant monoclone cell line HepG2/mdr1. Chin Med J 120: 703-707, 2007.

10. Li H, Cao HF, Wan J, Li Y, Zhu ML and Zhao P: Growth inhibitory effect of wild-type Kras2 gene on a colonic adenocarcinoma cell line. World J Gastroenterol 13: 934-938, 2007.

11. Sambrook J and Russell DW: Molecular Cloning: A Laboratory Manual. 3rd edition. Translated by Huang P. Science Press, Beijing, 2002.

12. Li K, Wang L, Cheng J, et al: Interaction between hepatitis $\mathrm{C}$ virus core protein and translin protein - a possible molecular mechanism for hepatocellular carcinoma and lymphoma caused by hepatitis C virus. World J Gastroenterol 9: 300-303, 2003.

13. Lu YY, Wang L, Liu Y, et al: Interaction between hepatitis b virus e antigen and the metal sulfur protein. Jie Fang Jun Yi Xue Za Zhi 28: 451-453, 2003 (In Chinese).

14. Cheng EH, Sheiko TV, Fisher JK, Craigen WJ and Korsmeyer SJ: VDAC2 inhibits BAK activation and mitochondrial apoptosis. Science 301: 513-517, 2003.

15. Karolchik D, Baertsch R, Diekhans M, et al; University of California Santa Cruz: The UCSC Genome Browser Database. Nucleic Acids Res 31: 51-54, 2003.

16. Rajasekaran S, Thapar V, Dave $\mathrm{H}$ and Huang $\mathrm{CH}$ : Randomized and parallel algorithms for distance matrix calculations in multiple sequence alignment. J Clin Monit Comput 19: 351-359, 2005.

17. Stocker RJ: The asialoglycoprotein receptors: relationships between structure, function and expression. Physiol Rev 75: 591-609, 1995.

18. Hajoui O, Martin S and Alvarez F: Study of antigenic sites on the asialoglycoprotein receptor recognized by autoantibodies. Clin Exp Immunol 113: 339-345, 1998.

19. Spring $P$, Nakashima T, Frederick M, Henderson $Y$ and Clayman G: Identification and cDNA cloning of headpin, a novel differentially expressed serpin that maps to chromosome 18q. Biochem Biophys Res Commun 264: 299-304, 1999.

20. Montrminy MR and Bilezikjan LM: Binding of a nuclear protein to the cyclic-AMP response element of the somatostatin gene. Nature 328: 175-178, 1987.

21. Lundblad M, Andersson M, Winkler C, Kirik D, Wierup N and Cenci MA: Pharmacological validation of behavioural measures of akinesia and dyskinesia in a rat model of Parkinson's disease. Eur J Neurosci 15: 120-132, 2002.

22. Isoda K, Kamezawa Y, Ayaori M, Kusuhara M, Tada N and Ohsuzu F: Osteopontin transgenic mice fed a high-cholesterol diet develop early fatty-streak lesions. Circulation 107: 679-681, 2003.

23. Abba MC, Laguens RM, Dulout FN and Golijow CD: The c-myc activation in cervical carcinomas and HPV 16 infections. Mutat Res 557: 151-158, 2004.

24. Tseng WF, Huang SS and Huang JS: LRP-1/TbetaR-V mediates TGF-betal-induced growth inhibition in CHO cells. FEBS Lett 562: 71-78, 2004

25. Ren F, Jin HY. Guo XH, et al: Erokaryotic expression and clinical application of X gene of hepatitis B virus. Wei Chang Bing Xue He Gan Bing Xue Za Zhi 15: 239-241, 2006 (In Chinese). 